\title{
Modified low regularity well-posedness for the one-dimensional Dirac-Klein-Gordon system
}

\author{
Hartmut Pecher
}

\begin{abstract}
The 1D Cauchy problem for the Dirac-Klein-Gordon system is shown to be locally well-posed for low regularity Dirac data in $\overline{H^{s, p}}$ and wave data in $\widehat{H^{r, p}} \times \widehat{H^{r-1}, p}$ for $1<p \leq 2$ under certain assumptions on the parameters $\mathrm{r}$ and $\mathrm{s}$, where $\|f\|_{\widehat{H^{s, p}}}:=\left\|\langle\xi\rangle^{s} \widehat{f}\right\|_{L^{p^{\prime}}}$, generalizing the results for $p=2$ by Selberg and Tesfahun. Especially we are able to improve the results from the scaling point of view with respect to the Dirac part.
\end{abstract}

Mathematics Subject Classification (2000). 35Q40, 35L70.

Keywords: Dirac - Klein - Gordon system, well-posedness, Fourier restriction norm method

\section{Introduction}

Consider the Cauchy problem for the Dirac - Klein - Gordon equations in one space dimension

$$
\begin{aligned}
-i \beta \frac{\partial}{\partial t} \psi+i \alpha \beta \frac{\partial}{\partial x} \psi+M \psi & =g \phi \psi \\
\frac{\partial^{2}}{\partial t^{2}} \phi-\frac{\partial^{2}}{\partial x^{2}} \phi+m^{2} \phi & =\langle\beta \psi, \psi\rangle_{\mathbf{C}^{2}}
\end{aligned}
$$

with initial data

$$
\psi(x, 0)=\psi_{0}(x), \phi(x, 0)=\phi_{0}(x), \frac{\partial \phi}{\partial t}(x, 0)=\phi_{1}(x) .
$$

Here $\psi$ is a two-spinor field, i.e. $\psi$ has values in $\mathbf{C}^{2}$, and $\phi$ is a real-valued function. $\alpha$ and $\beta$ are hermitian $(2 \times 2)$-matrices, which fulfill $\alpha^{2}=\beta^{2}=I, \alpha \beta+\beta \alpha=0$, e.g. we can choose $\alpha=\left(\begin{array}{ll}0 & 1 \\ 1 & 0\end{array}\right), \beta=\left(\begin{array}{ll}1 & 0 \\ 0 & -1\end{array}\right) . M, m$ and $g$ are real constants.

We are interested in local low regularity solutions. This problem was considered for data in $L^{2}$-based Sobolev spaces first by Chadam and Glassey [C], [CG] who proved global well-posedness for data $\psi_{0} \in H^{1}, \phi_{0} \in H^{1}, \phi_{1} \in L^{2}$. This result was improved by Bournaveas [B] (cf. also Fang $[\mathrm{F}]$ ) who showed the same results for data $\psi_{0} \in L^{2}, \phi_{0} \in H^{1}, \phi_{1} \in L^{2}$. Local well-posedness was shown by Fang [F1] 
for data $\psi_{0} \in H^{-\frac{1}{4}+\epsilon}, \phi_{0} \in H^{\frac{1}{2}+\delta}, \phi_{1} \in H^{-\frac{1}{2}+\delta}$ and $0<\epsilon \leq \frac{1}{4}, 0<\delta \leq 2 \epsilon$. Bournaveas and Gibbeson [BG] proved global well-posedness for $\psi_{0} \in L^{2}, \phi_{0} \in H^{k}$, $\phi_{1} \in H^{k-1}$ for $\frac{1}{4} \leq k<\frac{1}{2}$. The best known local well-posedness result for $L^{2}$ based Sobolev spaces, namely $\psi_{0} \in H^{s}, \phi_{0} \in H^{r}, \phi_{1} \in H^{r-1}$ was given by Selberg and Tesfahun [ST] who assumed $s>-1 / 4, r>0,|s| \leq r \leq 1+s$. This result was also shown to be optimal within the used method, namely the Bougain Klainerman - Machedon Fourier restriction norm method. This also improved earlier results of Machihara $[\mathrm{M}]$ and the author $[\mathrm{P}]$. The most recent results of $[\mathrm{ST}]$ and $[\mathrm{P}]$ were obtained using the null structure of $\langle\beta \psi, \psi\rangle$ of the wave part, which is also hidden (by a duality argument) in the Dirac part of the system. This fact was first detected by d'Ancona, Foschi and Selberg [AFS], who showed local well-posedness for data $\psi_{0} \in H^{\epsilon}, \phi_{0} \in H^{\frac{1}{2}+\epsilon}, \phi_{1} \in H^{-\frac{1}{2}+\epsilon}$ (for $\epsilon>0$ ) in the $(3+1)$-dimensional case, being arbitrarily close to the minimal regularity predicted by scaling $(\epsilon=0)$. In contrast to this $(3+1)$-dimensional result the best result in one space dimension by $[\mathrm{ST}]$ mentioned above is far away from the regularity predicted by scaling, namely $\psi_{0} \in H^{-1}, \phi_{0} \in H^{-1 / 2}, \phi_{1} \in H^{-3 / 2}$.

The aim of the present paper is to close this gap as far as possible. In order to achieve this we leave the $H^{s}$-scale of the data spaces. This was suggested for nonlinear Schrödinger equations by Cazenave, Varga, and Vilela [CVV] and Vargas and Vega [VV]. This method, a modified Fourier restriction norm method, was systematically introduced by Grünrock in [G1], where he applied it to the modified $\mathrm{KdV}$-equation and in [G2], where he was able to show local well-posedness in the case of the cubic nonlinear Schrödinger equation $i u_{t}+u_{x x}+|u|^{2} u=0, u(0)=u_{0}$, !! for data $u_{0} \in \widehat{H^{s, p}}$, where

$$
\left\|u_{0}\right\|_{\widehat{H^{s, p}}}:=\left\|\langle\xi\rangle^{s} \widehat{u_{0}}\right\|_{H_{\xi}^{p^{\prime}}}
$$

$1 / p+1 / p^{\prime}=1$, if $s \geq 0$ and $1<p<\infty$, and global well-posedness for $2 \geq p \geq 5 / 3$, $u_{0} \in \widehat{H^{0, p}}$.

In the present paper we prove local well-posedness for the Dirac-KleinGordon system with data $\psi_{0} \in \widehat{H^{s, p}}, \phi_{0} \in \widehat{H^{r, p}}, \phi_{1} \in \widehat{H^{r-1}, p}$ for $1<p \leq 2$ under suitable assumptions on $\mathrm{s}$ and $\mathrm{r}$. These results allow to improve the $L^{2}$ based results from the scaling point of view. More precisely we need the following conditions:

$$
s>-\frac{1}{2}+\frac{1}{2 p}, r \geq|s|, r>\frac{2}{p}-1, r \leq 1+s,
$$

which reduce to the results of $[\mathrm{ST}]$ in the case $p=2$. Especially we are able to choose $p=1+, s=0, r=1$, leading to $\psi_{0} \in \widehat{H^{0,1}}+$, which scales like $H^{-\frac{1}{2}+, 2}$ and to $\left(\phi_{0}, \phi_{1}\right) \in \widehat{H^{1,1}}+\times \widehat{H^{0,1}+}$, which scales like $H^{\frac{1}{2}+, 2} \times H^{-\frac{1}{2}+, 2}$. Thus the result by [ST],[P] in the case $p=2$, namely $\psi_{0} \in H^{-\frac{1}{4}+\epsilon, 2},\left(\phi_{0}, \phi_{1}\right) \in H^{\frac{1}{4}-\epsilon, 2} \times H^{-\frac{3}{4}-\epsilon, 2}$, $\frac{1}{4}>\epsilon>0$, is improved for the Dirac part at the expense of weakening the result for the wave part. 
This paper is organized as follows: We diagonalize the system like [ST] by using the projections $P_{ \pm}$onto the eigenspaces of $-i \alpha \frac{\partial}{\partial x}$ and splitting $\psi$ as a sum $P_{+} \psi+P_{-} \psi$. We also split $\phi$ as a sum $\phi=\phi_{+}+\phi_{-}$, where the half waves $\phi_{+}$and $\phi_{-}$are defined in the usual way. Then we analyze the components of the nonlinearity $\left\langle\beta \psi, \psi^{\prime}\right\rangle$, namely $\left\langle\beta P_{ \pm} \psi, P_{ \pm} \psi^{\prime}\right\rangle$ for all combinations of signs. It turns out that $\left\langle\beta P_{+} \psi, P_{-} \psi^{\prime}\right\rangle$ and $\left\langle\beta P_{-} \psi, P_{+} \psi^{\prime}\right\rangle$ vanish. Then we examine which bilinear estimates for the nonlinear terms are necessary for local well-posedness in the framework of the $X_{p}^{l, b}$-spaces (for a definition cf. (5),(6)). These are proven in Proposition 1.1. The results are summarized in Theorem 1.1 and the Remark to Theorem 1.1.

We recall the modified Fourier restriction norm method in the following. For details we refer to the paper of A. Grünrock (cf. [G1], Chapter 2). Our solution spaces are the Banach spaces

$$
X_{p}^{l, b}:=\left\{f \in \mathcal{S}^{\prime}\left(\mathbf{R}^{2}\right):\|f\|_{X_{p}^{l, b}}<\infty\right\}
$$

where $l, b \in \mathbf{R}, 1<p<\infty, 1 / p+1 / p^{\prime}=1$ and

$$
\|f\|_{X_{p}^{l, b}}:=\left(\int d \xi d \tau\langle\xi\rangle^{l p^{\prime}}\langle\tau+\phi(\xi)\rangle^{b p^{\prime}}|\hat{f}(\xi, \tau)|^{p^{\prime}}\right)^{1 / p^{\prime}}
$$

where $\phi: \mathbf{R} \rightarrow \mathbf{R}$ is a given smooth function of polynomial growth. We denote by $\tilde{f}$ or $\mathcal{F} f$ the Fourier transform with respect to space and time. The dual space of $X_{p}^{l, b}$ is $X_{p^{\prime}}^{-l,-b}$, and the Schwartz space is dense in $X_{p}^{l, b}$. We have $\left(X_{p_{0}}^{l_{0}, b_{0}}, X_{p_{1}}^{l_{1}, b_{1}}\right)_{[\Theta]}=X_{p}^{l, b}$, where $[\Theta]$ denotes the complex interpolation method, and for $l_{0}, l_{1}, b_{0}, b_{1} \in \mathbf{R}, 1<p_{0}, p_{1} \leq \infty, \Theta \in[0,1]$ we have $l=(1-\Theta) l_{0}+\Theta l_{1}$, $b=(1-\Theta) b_{0}+\Theta b_{1}, \frac{1}{p}=\frac{1-\Theta}{p_{0}}+\frac{\Theta}{p_{1}}$. The embedding $X_{p}^{l, b} \subset C^{0}\left(\mathbf{R}, \widehat{H^{l, p}}\right)$ is true for $b>1 / p$ (recall the definition (4)). We have

$$
\|f\|_{X_{p}^{l, b}}=\left(\int d \xi d \tau\langle\xi\rangle^{l p^{\prime}}\langle\tau\rangle^{b p^{\prime}}\left|\mathcal{F}\left(U_{\phi}(-\cdot) f\right)(\xi, \tau)\right|^{p^{\prime}}\right)^{1 / p^{\prime}}
$$

Here $U_{\phi}(t):=e^{-i t \phi\left(-i \partial_{x}\right)}$. For any $\psi \in C_{0}^{\infty}\left(\mathbf{R}_{t}\right)$ one has

$$
\left\|\psi U_{\phi}(t) u_{0}\right\|_{X_{p}^{l, b}} \leq c_{\psi}\left\|u_{0}\right\|_{\widehat{H^{l, p}}}
$$

If $v$ is a solution of the inhomogeneous problem

$$
i v_{t}-\phi\left(-i \partial_{x}\right) v=F, v(0)=0
$$

and $\psi \in C_{0}^{\infty}\left(\mathbf{R}_{t}\right)$ with supp $\psi \subset(-2,2), \psi \equiv 1$ on $[-1,1], \psi(t)=\psi(-t), \psi(t) \geq 0$, $\psi_{\delta}(t):=\psi\left(\frac{t}{\delta}\right), 0<\delta \leq 1$, we have for $1<p<\infty, b^{\prime}+1 \geq b \geq 0 \geq b^{\prime}>-1 / p^{\prime}$ :

$$
\left\|\psi_{\delta} v\right\|_{X_{p}^{l, b}} \leq c \delta^{1+b^{\prime}-b}\|F\|_{X_{p}^{l, b^{\prime}}}
$$


We also use the localized spaces

$$
X_{p}^{l, b}[0, T]:=\left\{f=\bar{f}_{\mid[0, T] \times \mathbf{R}}: \bar{f} \in X_{p}^{l, b}\right\},
$$

where

$$
\|f\|_{X_{p}^{l, b}[0, T]}:=\inf \left\{\|\bar{f}\|_{X_{p}^{l, b}}: f=\bar{f}_{\mid[0, T] \times \mathbf{R}}\right\} .
$$

For the case $\phi(\xi)= \pm \xi$ we use the notation $X_{ \pm p}^{l, b}$ and $\|\psi\|_{X_{ \pm p}^{l, b}}=\|\langle\xi\rangle^{l}\langle\tau \pm$ $\xi\rangle^{b} \tilde{\psi}(\xi, \tau) \|_{L_{\xi \tau}^{p^{\prime}}}$, whereas for the case $\phi(\xi)= \pm|\xi|$ we denote the space by $Y_{ \pm p}^{l, b}$ and $\|\psi\|_{Y_{ \pm p}^{l, b}}=\left\|\langle\xi\rangle^{l}\langle\tau \pm|\xi|\rangle^{b} \tilde{\psi}(\xi, \tau)\right\|_{L_{\xi \tau}^{p^{\prime}}}$

Especially we use [G1], Theorem 2.3, which we repeat for convenience.

Theorem 0.1. Consider the Cauchy problem

$$
i u_{t}-\phi\left(-i \partial_{x}\right) u=N(u) \quad, \quad u(0)=u_{0} \in \widehat{H^{s, p}},
$$

where $N$ is a nonlinear function of $u$ and its spatial derivatives. Assume for given $s \in \mathbf{R}, 1<p<\infty, \alpha \geq 1$ there exist $b>1 / p, b-1<b^{\prime} \leq 0$ such that the estimates

and

$$
\|N(u)\|_{X_{p}^{s, b^{\prime}}} \leq c\|u\|_{X_{p}^{s, b}}^{\alpha}
$$

$$
\|N(u)-N(v)\|_{X_{p}^{s, b^{\prime}}} \leq c\left(\|u\|_{X_{p}^{s, b}}^{\alpha-1}+\|v\|_{X_{p}^{s, b}}^{\alpha-1}\right)\|u-v\|_{X_{p}^{s, b}}
$$

are valid. Then there exist $T=T\left(\left\|u_{0}\right\|_{\widehat{H^{s, p}}}\right)>0$ and a unique solution $u \in$ $X_{p}^{s, b}[0, T]$ of (7). This solution belongs to $C^{0}\left([0, T], \widehat{H^{s, p}}\right)$, and the mapping $u_{0} \mapsto$ $u, \widehat{H^{s, p}} \rightarrow X_{p}^{s, b}\left(0, T_{0}\right)$ is locally Lipschitz continuous for any $T_{0}<T$.

We use the notation $\langle\lambda\rangle:=\left(1+\lambda^{2}\right)^{1 / 2}$, and $a \pm$ to denote a number slightly larger (resp., smaller) than $a$.

\section{Local well-posedness}

First we transform our system $(1),(2)$ into a first order system (in t) in diagonal form.

Multiplying the Dirac equations by $\beta$ leads to

$$
\begin{aligned}
-i \frac{\partial}{\partial t} \psi-i \alpha \frac{\partial}{\partial x} \psi+M \beta \psi & =g \phi \beta \psi \\
\frac{\partial^{2}}{\partial t^{2}} \phi-\frac{\partial^{2}}{\partial x^{2}} \phi+m^{2} \phi & =\langle\beta \psi, \psi\rangle_{\mathbf{C}^{2}} .
\end{aligned}
$$

Following the paper of Selberg and Tesfahun we diagonalize the system by defining the projections

$$
P_{ \pm}:=\frac{1}{2}\left(\begin{array}{cc}
1 & \pm 1 \\
\pm 1 & 1
\end{array}\right) .
$$


Then we have $\psi=\psi_{+}+\psi_{-}$with $\psi_{ \pm}:=P_{ \pm} \psi$. Using the identities $\alpha=P_{+}-P_{-}$, $P_{ \pm}^{2}=P_{ \pm}$and $P_{ \pm} P_{\mp}=0$ we get by application of $P_{ \pm}$to the Dirac equation

$$
\begin{aligned}
P_{ \pm}\left(-i \frac{\partial}{\partial t} \psi-i \alpha \frac{\partial}{\partial x} \psi\right) & =-i \frac{\partial}{\partial t} P_{ \pm} \psi-i \frac{\partial}{\partial x} P_{ \pm}\left(P_{+}-P_{-}\right) \psi \\
& =-i \frac{\partial}{\partial t} \psi_{ \pm} \mp i \frac{\partial}{\partial x} \psi_{ \pm}
\end{aligned}
$$

and thus the Dirac equations are transformed into

$$
\begin{aligned}
\left(-i \frac{\partial}{\partial t} \mp i \frac{\partial}{\partial x}\right) \psi_{ \pm} & =-M \beta P_{\mp}\left(\psi_{+}+\psi_{-}\right)+g P_{ \pm}(\phi \beta \psi) \\
& =-M \beta \psi_{\mp}+g P_{ \pm}\left(\phi \beta\left(\psi_{+}+\psi_{-}\right)\right),
\end{aligned}
$$

where we also used $P_{ \pm} \beta=\beta P_{\mp}$. We also split the function $\phi$ into the sum $\phi=\frac{1}{2}\left(\phi_{+}+\phi_{-}\right)$, where

$$
\phi_{ \pm}:=\phi \pm i A^{-\frac{1}{2}} \frac{\partial \phi}{\partial t} \quad, \quad A:=-\frac{\partial^{2}}{\partial x^{2}}+m^{2} .
$$

Here we assume $m>0$ and in fact $m=1$. Otherwise we artificially add a term $\left(1-m^{2}\right) \phi$ on both sides of the equation at the expense of having an additional linear term $c_{0} \phi$ in the inhomogeneous part which can easily be taken care of. We easily calculate

$$
\left(i \frac{\partial}{\partial t} \mp A^{\frac{1}{2}}\right) \phi_{ \pm}=\mp A^{-\frac{1}{2}}\left(\langle\beta \psi, \psi\rangle_{\mathbf{C}^{2}}+c_{0} \phi\right) .
$$

The initial conditions are transformed into

$$
\psi_{ \pm}(0, x)=P_{ \pm} \psi_{0}(x) \quad, \quad \phi_{ \pm}(0, x)=\phi_{0}(x) \pm i A^{-\frac{1}{2}} \phi_{1}(x) .
$$

The following (slightly modified) system of integral equations belongs to our Cauchy problem (8),(9),(10), where $U_{ \pm}(t)$ denotes the evolution operator of the equation $\left(\frac{\partial}{\partial t} \pm \frac{\partial}{\partial x}\right) u=0$.

$$
\begin{aligned}
\psi_{ \pm}(t)= & U_{ \pm}(t) \psi_{ \pm}(0)-i g \int_{0}^{t} U_{ \pm}(t-s) P_{ \pm}\left(\frac { 1 } { 2 } ( \phi _ { + } ( s ) + \phi _ { - } ( s ) ) \beta \left(P_{+} \psi_{+}(s)\right.\right. \\
+ & \left.\left.P_{-} \psi_{-}(s)\right)\right) d s+i M \int_{0}^{t} U_{ \pm}(t-s) \beta P_{\mp} \psi_{\mp}(s) d s \\
\phi_{ \pm}(t)= & e^{\mp i t A^{\frac{1}{2}}} \phi_{ \pm}(0) \\
& \pm i \int_{0}^{t} e^{\mp i(t-s) A^{\frac{1}{2}}} A^{-\frac{1}{2}}\left\langle\beta\left(P_{+} \psi_{+}(s)+P_{-} \psi_{-}(s)\right), P_{+} \psi_{+}(s)\right. \\
& \left.+P_{-} \psi_{-}(s)\right\rangle d s \pm i c_{0} \int_{0}^{t} e^{\mp i(t-s) A^{\frac{1}{2}}} A^{-\frac{1}{2}}\left(\phi_{+}(s)+\phi_{-}(s)\right) d s
\end{aligned}
$$


We remark that any solution of this system automatically fulfills $P_{ \pm} \psi_{ \pm}=\psi_{ \pm}$, because applying $P_{ \pm}$to the right hand side of the equations for $\psi_{ \pm}$gives $P_{ \pm} \psi_{ \pm}(0)=P_{ \pm} P_{ \pm} \psi_{0}=P_{ \pm} \psi_{0}=\psi_{ \pm}(0)$, and the integral terms also remain unchanged, because $P_{ \pm}^{2}=P_{ \pm}$and $P_{ \pm} \beta P_{\mp} \psi_{\mp}(s)=\beta P_{\mp} \psi_{\mp}(s)$. Thus $P_{ \pm} \psi_{ \pm}$can be replaced by $\psi_{ \pm}$on the right hand sides, thus the system of integral equations reduces exactly to the one belonging to our Cauchy problem $(8),(9),(10)$.

Let now data be given with

$$
\psi_{0} \in \widehat{H^{s, p}}, \phi_{0} \in \widehat{H^{r, p}}, \phi_{1} \in \widehat{H^{r-1}, p}
$$

This implies $\psi_{ \pm}(0) \in \widehat{H^{s, p}}$ and $\phi_{ \pm}(0) \in \widehat{H^{r, p}}$. In order to construct a solution of the integral equations for $t \in[0, T]$ with $\psi_{ \pm} \in X_{ \pm p}^{s, \sigma}[0, T]$ and $\phi_{ \pm} \in Y_{ \pm p}^{r, \rho}[0, T]$, where $1>\sigma, \rho>1 / p$, we may apply Theorem 0.1 , because its generalization from the case of a single equation to a system is evident. Thus we only have to show the following estimates for the nonlinearities.

Concerning (11) we need

$$
\left\|P_{ \pm}\left(\phi \beta P_{[ \pm]} \psi\right)\right\|_{X_{ \pm p}^{s, \sigma-1+\epsilon}} \leq c\|\phi\|_{Y_{+p}^{r, \rho}}\|\psi\|_{X_{[ \pm] p}^{s, \sigma}}
$$

and the same estimates with $Y_{+p}^{r, \rho}$ replaced by $Y_{-p}^{r, \rho} \cdot[ \pm]$ denotes a sign independent of \pm . By duality this is equivalent to

$$
\left|\iint\left\langle P_{ \pm}\left(\phi \beta P_{[ \pm]} \psi\right), \psi^{\prime}\right\rangle d x d t\right| \leq c\|\phi\|_{Y_{+p}^{r, \rho}}\|\psi\|_{X_{[ \pm] p}^{s, \sigma}}\left\|\psi^{\prime}\right\|_{X_{ \pm p^{\prime}}^{-s, 1-\sigma-\epsilon}} .
$$

The left hand side equals

$$
\left|\iint \phi\left\langle\beta P_{[ \pm]} \psi, P_{ \pm} \psi^{\prime}\right\rangle d x d t\right|
$$

which can be estimated by

$$
\|\phi\|_{Y_{+p}^{r, \rho}}\left\|\left\langle\beta P_{[ \pm]}(D) \psi, P_{ \pm} \psi^{\prime}\right\rangle\right\|_{Y_{+p^{\prime}}^{-r,-\rho}} .
$$

Thus (13) is fulfilled if

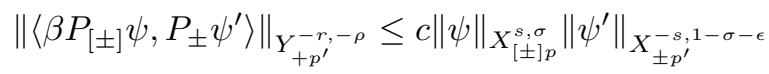

Concerning (12) we have to show

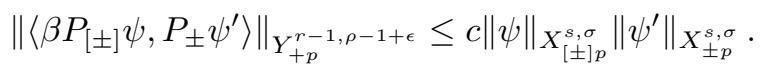

We also need the same estimates with $Y_{+}$replaced by $Y_{-}$. 
The linear terms in the integral equations can easily be treated as follows: Let $\varphi$ be a $t$-dependent $C^{\infty}$ - function with $\varphi=1$ on $[0, T]$ and $\operatorname{supp} \varphi \subset[0,2 T]$. Then, with $J^{s}$ being the multiplier with symbol $\langle\xi\rangle^{s}$, and using the embedding (42) we have:

$$
\begin{aligned}
\left\|\psi_{ \pm}\right\|_{X_{ \pm p}^{s, \sigma-1+\epsilon}[0, T]} & \leq\left\|\varphi \overline{\psi_{ \pm}}\right\|_{X_{ \pm p}^{s, \sigma-1+\epsilon}} \leq\left\|\varphi \overline{\psi_{ \pm}}\right\|_{X_{ \pm p}^{s, 0}} \\
& =\left\|\varphi J^{s} \overline{\psi_{ \pm}}\right\|_{\widehat{L_{x t}^{p}}} \leq\|\varphi\|_{\widehat{L_{t}^{p}}}\left\|J^{s} \overline{\psi_{ \pm}}\right\|_{\widehat{L_{x}^{p}}\left(\widehat{L_{t}^{\infty}}\right)} \\
& \leq\|\varphi\|_{\widehat{L_{t}^{p}}}\left\|J^{s} \overline{\psi_{ \pm}}\right\|_{X_{ \pm p}^{0, \sigma}} \leq c T^{1 / p}\left\|\overline{\psi_{ \pm}}\right\|_{X_{ \pm p}^{s, \sigma}}
\end{aligned}
$$

for any $\overline{\psi_{ \pm}}$with $\overline{\psi_{ \pm} \mid[0, T]}=\psi_{ \pm}$. Here we used Hausdorff-Young to estimate $\|\varphi\|_{\widehat{L_{t}^{p}}}$ by $c T^{1 / p}$. Thus:

$$
\left\|\psi_{ \pm}\right\|_{X_{ \pm p}^{s, \sigma-1+\epsilon}[0, T]} \leq c T^{1 / p}\left\|\psi_{ \pm}\right\|_{X_{ \pm p}^{s, \sigma}[0, T]}
$$

and similarly

$$
\left\|A^{-1 / 2} \phi_{ \pm}\right\|_{Y_{ \pm p}^{0, \rho-1+\epsilon}[0, T]} \leq c T^{1 / p}\left\|\phi_{ \pm}\right\|_{Y_{ \pm p}^{r, \rho}[0, T]} .
$$

The bilinear form has a null structure, we namely have

$$
\left\langle\beta P_{ \pm} \psi, P_{ \pm} \psi^{\prime}\right\rangle=\left\langle P_{\mp} \beta \psi, P_{ \pm} \psi^{\prime}\right\rangle=0
$$

so that in order to prove (14) and (15) it remains to show

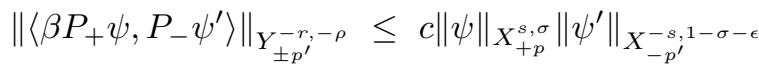

$$
\begin{aligned}
& \left\|\left\langle\beta P_{-} \psi, P_{+} \psi^{\prime}\right\rangle\right\|_{Y_{ \pm p^{\prime}}^{-r, \rho}} \leq c\|\psi\|_{X_{-p}^{s, \sigma}}\left\|\psi^{\prime}\right\|_{X_{+p^{\prime}}^{-s, 1-\sigma-\epsilon}} \\
& \left\|\left\langle\beta P_{+} \psi, P_{-} \psi^{\prime}\right\rangle\right\|_{Y_{ \pm p}^{r-1, \rho-1+\epsilon}} \leq c\|\psi\|_{X_{+p}^{s, \sigma}}\left\|\psi^{\prime}\right\|_{X_{-p}^{s, \sigma}} \\
& \left\|\left\langle\beta P_{-} \psi, P_{+} \psi^{\prime}\right\rangle\right\|_{Y_{ \pm p}^{r-1, \rho-1+\epsilon}} \leq c\|\psi\|_{X_{-p}^{s, \sigma}}\left\|\psi^{\prime}\right\|_{X_{+p}^{s, \sigma}} .
\end{aligned}
$$

In order to prove (18) we have to show

$$
\left|\iint\left\langle\beta P_{+} \psi, P_{-} \psi^{\prime}\right\rangle \bar{\phi} d x d t\right| \leq c\|\phi\|_{Y_{ \pm p^{\prime}}^{1-r, 1-\rho-\epsilon}}\|\psi\|_{X_{+p}^{s, \sigma}}\left\|\psi^{\prime}\right\|_{X_{-p}^{s, \sigma}} .
$$

The left hand side equals

$$
\left|\iint_{*}\left\langle\beta P_{+} \tilde{\psi}\left(\xi_{1}, \tau_{1}\right), P_{-} \tilde{\psi}^{\prime}\left(-\xi_{2},-\tau_{2}\right)\right\rangle \overline{\tilde{\phi}}(\xi, \tau) d \xi_{1} d \xi_{2} d \tau_{1} d \tau_{2}\right| \text {, }
$$

where $^{*}$ denotes the region $\xi_{1}+\xi_{2}=\xi, \tau_{1}+\tau_{2}=\tau$.

Defining now

$$
\begin{aligned}
\tilde{v}_{1}\left(\xi_{1}, \tau_{1}\right) & :=\left\langle\xi_{1}\right\rangle^{s}\left\langle\tau_{1}+\xi_{1}\right\rangle^{\sigma} \tilde{\psi}\left(\xi_{1}, \tau_{1}\right) \\
\tilde{v}_{2}\left(\xi_{2}, \tau_{2}\right) & :=\left\langle\xi_{2}\right\rangle^{s}\left\langle\tau_{2}-\xi_{2}\right\rangle^{\sigma} \tilde{\psi}^{\prime}\left(\xi_{2}, \tau_{2}\right) \\
\tilde{\varphi}(\xi, \tau) & :=\langle\xi\rangle^{1-r}\langle\tau \pm|\xi|\rangle^{1-\rho-\epsilon} \tilde{\phi}(\xi, \tau)
\end{aligned}
$$


we have

$$
\|\psi\|_{X_{+p}^{s, \sigma}}=\left\|v_{1}\right\|_{\widehat{L_{x t}^{p}}},\left\|\psi^{\prime}\right\|_{X_{-p}^{s, \sigma}}=\left\|v_{2}\right\|_{\widehat{L_{x t}^{p}}},\|\phi\|_{Y_{ \pm p^{\prime}}^{1-r, 1-\rho-\epsilon}}=\|\varphi\|_{\widehat{L_{x t}^{p^{\prime}}}} .
$$

Thus we have to show

$$
\begin{aligned}
& \iint_{*} \frac{\left|\tilde{v}_{1}\left(\xi_{1}, \tau_{1}\right) \tilde{v}_{2}\left(-\xi_{2},-\tau_{2}\right) \overline{\tilde{\varphi}}(\xi, \tau)\right|}{\left\langle\xi_{1}\right\rangle^{s}\left\langle\tau_{1}+\xi_{1}\right\rangle^{\sigma}\left\langle\xi_{2}\right\rangle^{s}\left\langle\tau_{2}-\xi_{2}\right\rangle^{\sigma}\langle\xi\rangle^{1-r}\langle\tau \pm|\xi|\rangle^{1-\rho-\epsilon}} d \xi_{1} d \xi_{2} d \tau_{1} d \tau_{2} \\
& \leq c\left\|v_{1}\right\|_{\widehat{L^{p}}}\left\|v_{2}\right\|_{\widehat{L^{p}}}\|\varphi\|_{\widehat{L^{p^{\prime}}}}
\end{aligned}
$$

Define $\sigma_{1}^{+}:=\tau_{1}+\xi_{1}, \sigma_{2}^{-}:=\tau_{2}-\xi_{2}, \sigma_{ \pm}:=\tau \pm|\xi|$.

Case $1\left|\sigma_{ \pm}\right|$dominant, i.e. $\left|\sigma_{ \pm}\right| \geq\left|\sigma_{1}^{+}\right|,\left|\sigma_{2}^{-}\right|$.

a: $\left|\xi_{1}\right|<<\left|\xi_{2}\right|\left(\Rightarrow|\xi| \sim\left|\xi_{2}\right|\right)$. (The case $\left|\xi_{2}\right|<<\left|\xi_{1}\right|$ can be treated similarly.) In this case we have by the algebraic inequality in Lemma 2.1 the estimate $\left\langle\sigma_{ \pm}\right\rangle \geq$ $c\left\langle\xi_{1}\right\rangle$, so that it remains to estimate (provided $\rho \leq 1-\epsilon$ ):

$$
\begin{aligned}
& \iint_{*} \frac{\left|\tilde{v}_{1}\left(\xi_{1}, \tau_{1}\right) \tilde{v}_{2}\left(-\xi_{2},-\tau_{2}\right) \bar{\varphi}(\xi, \tau)\right|}{\left\langle\xi_{1}\right\rangle^{s+1-\rho-\epsilon}\left\langle\sigma_{1}^{+}\right\rangle^{\sigma}\left\langle\xi_{2}\right\rangle^{s+1-r}\left\langle\sigma_{2}^{-}\right\rangle^{\sigma}} d \xi_{1} d \xi_{2} d \tau_{1} d \tau_{2} \\
& \quad \leq c \iint_{*} \frac{\left|\tilde{v}_{1}\left(\xi_{1}, \tau_{1}\right) \tilde{v}_{2}\left(-\xi_{2},-\tau_{2}\right) \tilde{\varphi}(\xi, \tau)\right|}{\left\langle\sigma_{1}^{+}\right\rangle^{\sigma}\left\langle\sigma_{2}^{-}\right\rangle^{\sigma}} d \xi_{1} d \xi_{2} d \tau_{1} d \tau_{2},
\end{aligned}
$$

where we made the assumptions

$$
s+1-\rho-\epsilon \geq 0
$$

and

$$
s+1-r \geq 0 .
$$

Using Corollary 2.1 below we get the desired bound.

b $\left|\xi_{1}\right| \sim\left|\xi_{2}\right|$

Using the estimate $\left\langle\sigma_{ \pm}\right\rangle \geq c\left\langle\xi_{1}\right\rangle$ again we arrive at

$$
\iint_{*} \frac{\left|\tilde{v}_{1}\left(\xi_{1}, \tau_{1}\right) \tilde{v}_{2}\left(-\xi_{2},-\tau_{2}\right) \overline{\tilde{\varphi}}(\xi, \tau)\right|}{\left\langle\xi_{1}\right\rangle^{2 s+1-\rho-\epsilon}\langle\xi\rangle^{1-r}\left\langle\sigma_{1}^{+}\right\rangle^{\sigma}\left\langle\sigma_{2}^{-}\right\rangle^{\sigma}} d \xi_{1} d \xi_{2} d \tau_{1} d \tau_{2} .
$$

If $r>1$ we further have $\langle\xi\rangle^{r-1} \leq c\left(\left\langle\xi_{1}\right\rangle^{r-1}+\left\langle\xi_{2}\right\rangle^{r-1}\right)$ and get the bound

$$
\iint_{*} \frac{\left|\tilde{v}_{1}\left(\xi_{1}, \tau_{1}\right) \tilde{v}_{2}\left(-\xi_{2},-\tau_{2}\right) \overline{\tilde{\varphi}}(\xi, \tau)\right|}{\left\langle\xi_{1}\right\rangle^{2 s+1-\rho-\epsilon+1-r}\left\langle\sigma_{1}^{+}\right\rangle^{\sigma}\left\langle\sigma_{2}^{-}\right\rangle^{\sigma}} d \xi_{1} d \xi_{2} d \tau_{1} d \tau_{2} .
$$

Using again the bilinear estimate of Corollary 2.1 we get the desired bound provided

$$
2 s+1-\rho-\epsilon \geq 0
$$


and

$$
2 s+1-\rho-\epsilon+1-r \geq 0 .
$$

Case $2\left|\sigma_{1}^{+}\right|$dominant and $\left|\xi_{2}\right| \geq\left|\xi_{1}\right|$. (The other cases: $\left|\sigma_{2}^{-}\right|$dominant and/or $\left|\xi_{2}\right| \leq\left|\xi_{1}\right|$ are similar.)

Using the algebraic inequality $\left|\sigma_{1}^{+}\right| \geq c\left|\xi_{1}\right|$ we have to estimate

$$
\iint_{*} \frac{\left|\tilde{v}_{1}\left(\xi_{1}, \tau_{1}\right) \tilde{v}_{2}\left(-\xi_{2},-\tau_{2}\right) \overline{\tilde{\varphi}}(\xi, \tau)\right|}{\left\langle\xi_{1}\right\rangle^{s+\sigma}\left\langle\xi_{2}\right\rangle^{s}\left\langle\sigma_{2}^{-}\right\rangle^{\sigma}\langle\xi\rangle^{1-r}\left\langle\sigma_{ \pm}\right\rangle^{1-\rho-\epsilon}} d \xi_{1} d \xi_{2} d \tau_{1} d \tau_{2} .
$$

We apply Proposition 2.2 with $a=s+\sigma, b=s, c=1-r, \alpha=0, \beta=\sigma$, $\gamma=1-\rho-\epsilon$ and get the desired estimate provided

$$
\begin{aligned}
2 s+\sigma+1-r & >\frac{1}{p}+\frac{1}{p}+\frac{1}{p^{\prime}}-1=\frac{1}{p} \\
2 s+\sigma & \geq 0 \\
s+\sigma+1-r & \geq 0 \\
s+1-r & \geq 0 \\
\sigma+1-\rho-\epsilon & >\frac{1}{p}+\frac{1}{p}+\frac{1}{p^{\prime}}-1=\frac{1}{p} \\
\rho & \leq 1-\epsilon .
\end{aligned}
$$

We conclude that (18) (and similarly (19)) holds, if (20)-(29) are satisfied.

Next we have to prove (16). Similarly as before we have to show

$$
\begin{array}{r}
\iint_{*} \frac{\left|\tilde{v}_{1}\left(\xi_{1}, \tau_{1}\right) \tilde{v}_{2}\left(-\xi_{2},-\tau_{2}\right) \overline{\tilde{\varphi}}(\xi, \tau)\right|}{\left\langle\xi_{1}\right\rangle^{s}\left\langle\sigma_{1}^{+}\right\rangle^{\sigma}\left\langle\xi_{2}\right\rangle^{-s}\left\langle\sigma_{2}^{-}\right\rangle^{1-\sigma-\epsilon}\langle\xi\rangle^{r}\left\langle\sigma_{ \pm}\right\rangle^{\rho}} d \xi_{1} d \xi_{2} d \tau_{1} d \tau_{2} \\
\leq c\left\|v_{1}\right\|_{\widehat{L^{p}}}\left\|v_{2}\right\| \widehat{L^{p^{\prime}}}\|\varphi\|_{\widehat{L^{p}}} .
\end{array}
$$

We use the algebraic inequality of Lemma 2.1 and remark that $\min (\sigma, \rho, 1-\sigma-\epsilon)=$ $1-\sigma-\epsilon$, because $\sigma, \rho>1 / p>1 / 2$ for $1<p \leq 2$. Assuming

$$
\sigma \leq 1-\epsilon
$$

we can therefeore replace one of the expressions $\left\langle\sigma_{1}^{+}\right\rangle^{1-\sigma-\epsilon},\left\langle\sigma_{2}^{+}-\right\rangle^{1-\sigma-\epsilon}$ or $\left\langle\sigma_{ \pm}\right\rangle^{1-\sigma-\epsilon}$ by $\left\langle\xi_{1}\right\rangle^{1-\sigma-\epsilon}$ or $\left\langle\xi_{2}\right\rangle^{1-\sigma-\epsilon}$. In any case the conditions of Proposition 2.2 are satisfied to give the desired bound provided the following assumptions are made:

$$
\begin{aligned}
s-s+r+1-\sigma-\epsilon & >\frac{1}{p} \\
s-s+1-\sigma-\epsilon & \geq 0 \\
s+r & \geq 0 \\
-s+r & \geq 0 \\
s+1-\sigma-\epsilon+r & \geq 0 \\
-s+1-\sigma-\epsilon+r & \geq 0 .
\end{aligned}
$$


The sum of the exponents of the remaining $\sigma$-modules is $\rho+\sigma$ which has to be larger than $1 / p$. This is trivially satisfied.

(17) is proven completely analogously.

We summarize our results in the following

Proposition 1.1. Let $1<p \leq 2$. The inequalities (16),(17),(18),(19) are satisfied with suitable $1>\sigma, \rho>1 / p$, if the following conditions hold:

$$
\begin{aligned}
& s>-\frac{1}{2}+\frac{1}{2 p} \\
& r \leq 1+s \\
& r \geq|s| \\
& r>\frac{2}{p}-1 .
\end{aligned}
$$

Proof. We only have to check the conditions (20)-(36). Choose $\rho=\frac{1}{p}+\epsilon, \epsilon>0$ small. Then:

(20) is satisfied, because by use of (37) we have $s+1-\rho-\epsilon \frac{1}{2}-\frac{1}{2 p}-2 \epsilon>0$.

(21) is equivalent to (38).

(22) follows from (37): $2 s+1-\rho-\epsilon>0$.

(23): $2 s+1-\rho-\epsilon+1-r \geq s+1-\frac{1}{p}-2 \epsilon>\frac{1}{2}-\frac{1}{2 p} \geq 0$ by (38) and (37).

(25): $2 s+\sigma>-1+\frac{2}{p} \geq 0$ by (37) and $\sigma>1 / p$.

(26) is weaker than (27), which is equivalent to (38).

(28),(29),(30) are fulfilled, because $1>\sigma>\frac{1}{p}$ and $\rho<1$.

(32) is satisfied for $\sigma<1$.

(33) and (34) are equivalent to (39).

(35),(36): $\pm s+1-\sigma+r \geq \pm s+1-\sigma+|s| \geq 1-\sigma \geq 0$ by (39).

It remains to fulfill (24): $r<2 s+\sigma+1-\frac{1}{p}$ and (31): $r>\sigma-1+\frac{1}{p}+\epsilon$.

These conditions can be fulfilled with a suitable $1 / p<\sigma<1$, provided $r<$ $2 s+2-\frac{1}{p}$ and $r>\frac{2}{p}-1$. The last condition is (40), and the first one is weaker than (38) under assumption (37): $2 s+2-\frac{1}{p}>1+s \Longleftrightarrow s>-1+\frac{1}{p}$. This holds, if (37) is fulfilled.

Thus we have proven the following

Theorem 1.1. Assume $1<p \leq 2$ and (37)-(40). The Cauchy problem for the Dirac - Klein - Gordon system (1),(2),(3) with data

$$
\psi_{0} \in \widehat{H^{s, p}}, \phi_{0} \in \widehat{H^{r, p}}, \phi_{1} \in \widehat{H^{r-1}, p}
$$

is locally well-posed, i.e. there exists a unique local solution

$$
\psi=\psi_{+}+\psi_{-} \quad \text { with } \quad \psi_{ \pm} \in X_{ \pm p}^{s, \sigma}[0, T]
$$


and

$$
\phi=\frac{1}{2}\left(\phi_{+}+\phi_{-}\right), \phi_{t}=\frac{1}{2 i} A^{-\frac{1}{2}}\left(\phi_{+}-\phi_{-}\right) \quad \text { with } \quad \phi_{ \pm} \in Y_{ \pm p}^{r, \rho}[0, T],
$$

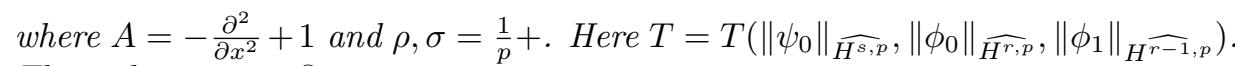
This solution satisfies

$$
\psi \in C^{0}\left([0, T], \widehat{H^{s, p}}\right), \phi \in C^{0}\left([0, T], \widehat{H^{r, p}}\right), \phi_{t} \in C^{0}\left([0, T], \widehat{H^{r-1}, p}\right),
$$

and the mapping data upon solution is locally Lipschitz continuous.

Remark From the scaling point of view the spaces $\widehat{H^{s, p}}$ behave like the Sobolev spaces $H^{s, p}$ and like $H^{\sigma, 2}$, where $\sigma=s+\frac{1}{2}-\frac{1}{p}$. Similarly $\widehat{H^{r, p}}$ behaves like $H^{\lambda, 2}$, where $\lambda=r+\frac{1}{2}-\frac{1}{p}$. This has the following consequences in view of our assumptions (37)-(40).

Minimizing $\sigma$ requires to take $s=-\frac{1}{2}+\frac{1}{2 p}+$, so that $\sigma_{\min }=-\frac{1}{2 p}+$, which is optimal for $p=1+$, namely $\sigma_{\text {min }}=-\frac{1}{2}+$. The corresponding $\lambda$ in the case $s=-\frac{1}{2}+\frac{1}{2 p}+$ is limited by the conditions (39) and (40), which require $r>\frac{2}{p}-1$ for $1<p \leq 5 / 3$, and $r>\frac{1}{2}-\frac{1}{2 p}-$ for $5 / 3<p \leq 2$. Thus $\lambda_{\text {min }}=\frac{1}{p}-\frac{1}{2}+$ for $1<p \leq 5 / 3$, and $\lambda_{\min }=1-\frac{3}{2 p}-$ for $5 / 3<p \leq 2$, especially for $p=1+$ : $\lambda_{\min }=\frac{1}{2}+$.

The minimal $\lambda$, however, is obtained for $s=0$ and $p=2$, namely $r=0+$, $p=2$, thus $\lambda=0+$.

Summarizing, we are able to improve the result from the scaling point of view for the Dirac part by leaving the case $p=2$, whereas for the wave part no improvement can be given.

\section{Appendix}

We use the notation $\|f\|_{\widehat{L_{x}^{p}}\left(\widehat{L_{t}^{q}}\right)}:=\|\tilde{f}\|_{L_{\xi}^{p^{\prime}}\left(L_{\tau}^{q^{\prime}}\right)}$, where $\tilde{f}$ is the Fourier transform with respect to space and time. First we prove an embedding theorem.

Proposition 2.1. Let $1<r, w_{1}, w_{2}, w_{3}<\infty$. Then the following embeddings hold:

$$
\begin{aligned}
X_{r}^{\frac{1}{r}+, 0} & \subset \widehat{L_{x}^{\infty}}\left(\widehat{L_{t}^{r}}\right) \\
X_{r}^{0, \frac{1}{r}+} & \subset \widehat{L_{x}^{r}}\left(\widehat{L_{t}^{\infty}}\right) \\
X_{r}^{\frac{1}{r}+, \frac{1}{r}+} & \subset \widehat{L_{x}^{\infty}}\left(\widehat{L_{t}^{\infty}}\right) \\
X_{w_{3}}^{\frac{1}{w_{1}}+, \frac{1}{w_{2}}}+ & \subset \widehat{L_{x}^{v_{1}}}\left(\widehat{L_{t}^{v_{2}}}\right)
\end{aligned}
$$

where $1 / v_{1}=1 / w_{3}-1 / w_{1}, 1 / v_{2}=1 / w_{3}-1 / w_{2}$. 
Proof. (41): Hölder's inequality gives

$$
\|u\|_{\widehat{L_{x}^{\infty}}\left(\widehat{L_{t}^{r}}\right)}=\int\left(\left(\int|\tilde{u}(\xi, \tau)|^{r^{\prime}} d \tau\right)^{1 / r^{\prime}}\langle\xi\rangle^{1 / r+}\right)\langle\xi\rangle^{-1 / r-} d \xi \leq c\|u\|_{X_{r}^{1 / r+, 0}} .
$$

(42): Similarly

$$
\begin{aligned}
\|u\|_{\widehat{L_{x}^{r}}\left(\widehat{L_{t}^{\infty}}\right)}= & \left(\int\left(\int|\tilde{u}(\xi, \tau)|\langle\tau+\phi(\xi)\rangle^{1 / r+}\langle\tau+\phi(\xi)\rangle^{-1 / r-} d \tau\right)^{r^{\prime}} d \xi\right)^{1 / r^{\prime}} \\
\leq & \left(\left(\int|\tilde{u}(\xi, \tau)|^{r^{\prime}}\langle\tau+\phi(\xi)\rangle^{r^{\prime}\left(\frac{1}{r}+\right)} d \tau\right)\right. \\
& \left.\left(\int\langle\tau+\phi(\xi)\rangle^{\left(-\frac{1}{r}-\right) r} d \tau\right)^{\frac{r^{\prime}}{r}} d \xi\right)^{1 / r^{\prime}} \leq c\|u\|_{X_{r}^{0,1 / r+}}
\end{aligned}
$$

(43): Using Hölder's inequality twice we get

$$
\begin{aligned}
\|u\|_{\widehat{L_{t}^{\infty}}\left(\widehat{L_{x}^{\infty}}\right)} & =\iint \mid \tilde{u}(\xi, \tau)\langle\tau+\phi(\xi)\rangle^{1 / r+}\langle\tau+\phi(\xi)\rangle^{-1 / r-} d \tau d \xi \\
& \leq c \int\left(\int\left|\tilde{u}(\xi, \tau)\langle\tau+\phi(\xi)\rangle^{1 / r+}\right|^{r^{\prime}} d \tau\right)^{1 / r^{\prime}}\langle\xi\rangle^{1 / r+}\langle\xi\rangle^{-1 / r-} d \xi \\
& \leq c\|u\|_{X_{r}^{1 / r+, 1 / r+}} .
\end{aligned}
$$

(44): In a first step we interpolate between (41) and (43) and get

$$
X_{r}^{1 / r+, 1 / r_{1}+} \subset \widehat{L_{x}^{\infty}}\left(\widehat{L_{t}^{r_{2}}}\right),
$$

where $1 / r_{2}=1 / r-1 / r_{1}$. Similarly, interpolation between (42) and (43) gives

$$
X_{r}^{1 / r_{1}+, 1 / r+} \subset \widehat{L_{x}^{r_{2}}}\left(\widehat{L_{t}^{\infty}}\right),
$$

where $1 / r_{2}=1 / r-1 / r_{1}$. Finally, interpolating between (45) and (46) gives the desired result.

We now use this proposition to prove a product law for $X_{r, \phi}^{s, b}$-spaces, belonging to the phase function $\phi$.

Proposition 2.2. Let $1<p, q, r<\infty, \alpha, \beta, \gamma \geq 0$. Assume $a+b+c>1 / p+$ $1 / q+1 / r^{\prime}-1, a+b \geq 0, a+c \geq 0, b+c \geq 0$ and $\alpha+\beta+\gamma>1 / p+1 / q+1 / r^{\prime}-1$. Then the following estimate holds:

$$
\|u v\|_{X_{r, \phi}^{-c,-\gamma}} \leq c\|u\|_{X_{p, \phi_{1}}^{a, \alpha}}\|v\|_{X_{q, \phi_{2}}^{b, \beta}} .
$$


Proof. Using $\left(X_{r, \phi}^{-c,-\gamma}\right)^{*}=X_{r^{\prime}, \phi}^{c, \gamma}$ we have to show

$$
\left|\iint_{*} \frac{\tilde{v}_{1}\left(\xi_{1}, \tau_{1}\right)}{\left\langle\xi_{1}\right\rangle^{a}\left\langle\sigma_{1}\right\rangle^{\alpha}} \frac{\tilde{v}_{2}\left(\xi_{2}, \tau_{2}\right)}{\left\langle\xi_{2}\right\rangle^{b}\left\langle\sigma_{2}\right\rangle^{\beta}} \frac{\tilde{\varphi}(\xi, \tau)}{\langle\xi\rangle^{c}\langle\sigma\rangle^{\gamma}} d \xi_{1} d \xi_{2} d \tau_{1} d \tau_{2}\right| \leq c\left\|v_{1}\right\|_{\widehat{L^{p}}}\left\|v_{2}\right\|_{\widehat{L^{q}}}\|\varphi\|_{\widehat{L^{r^{\prime}}}}
$$

where $\sigma_{i}=\tau_{i}+\phi_{i}\left(\xi_{i}\right), \sigma=\tau+\phi(\xi)$.

Assume $a, b, c \geq 0$ first. Applying Young's and Hölder's inequality we get a bound

$$
c\left\|J^{-a} \Lambda_{1}^{-\alpha} v_{1}\right\|_{\widehat{L_{x}^{p_{1}}}\left(\widehat{L_{t}^{p_{2}}}\right)}\left\|J^{-b} \Lambda_{2}^{-\beta} v_{2}\right\|_{\widehat{L_{x}^{q 1}}\left(\widehat{L_{t}^{q q_{2}}}\right)}\left\|J^{-c} \Lambda^{-\gamma} \varphi\right\|_{\widehat{L_{x}^{r 1}}\left(\widehat{L_{t}^{r r^{2}}}\right)}
$$

where $1 / p_{1}+1 / q_{1}+1 / r_{1}=1=1 / p_{2}+1 / q_{2}+1 / r_{2}$. Here $J$ and $\Lambda_{1}, \Lambda_{2}, \Lambda$ are the Fourier multipliers with symbols $\langle\xi\rangle$ and $\left\langle\sigma_{1}\right\rangle,\left\langle\sigma_{2}\right\rangle,\langle\sigma\rangle$, respectively. In order to get the desired bound we need the embeddings:

$$
X_{p, \phi_{1}}^{a, \alpha} \subset \widehat{L_{x}^{p_{1}}}\left(\widehat{L_{t}^{p_{2}}}\right), X_{q, \phi_{2}}^{b, \beta} \subset \widehat{L_{x}^{q_{1}}}\left(\widehat{L_{t}^{q_{2}}}\right), X_{r^{\prime}, \phi}^{c, \gamma} \subset \widehat{L_{x}^{r_{1}}}\left(\widehat{L_{t}^{r_{2}}}\right) .
$$

These embeddings hold by Proposition 2.1, if the following conditions are satisfied:

$$
\begin{array}{r}
1 / p_{1}>1 / p-a, 1 / p_{2}>1 / p-\alpha, 1 / q_{1}>1 / q-b \\
1 / q_{2}>1 / q-\beta, 1 / r_{1}>1 / r^{\prime}-c, 1 / r_{2}>1 / r^{\prime}-\gamma .
\end{array}
$$

These inequalities can obviously be fulfilled if $1=1 / p_{1}+1 / q_{1}+1 / r_{1}>1 / p+1 / q+$ $1 / r^{\prime}-(a+b+c) \Leftrightarrow a+b+c>1 / p+1 / q+1 / r^{\prime}-1$ and $1=1 / p_{2}+1 / q_{2}+1 / r_{2}>$ $1 / p+1 / q+1 / r^{\prime}-(\alpha+\beta+\gamma) \Leftrightarrow \alpha+\beta+\gamma>1 / p+1 / q+1 / r^{\prime}-1$.

Consider now the case, where not all the numbers $a, b, c$ are nonnegative. In view of our assumptions only one number is negative, $a<0$, say. Then $\left\langle\xi_{1}\right\rangle^{-a} \leq$ $c\left(\left\langle\xi_{2}\right\rangle^{-a}+\langle\xi\rangle^{-a}\right)$. Thus we only have to consider the integral with $(a, b, c)$ replaced by $(0, a+b, c)$ or $(0, b, a+c)$. All these numbers are nonnegative and their sum also fulfills our assumptions. This proves the general case.

Finally, we prove a bilinear estimate for free waves.

Proposition 2.3. If $u$ and $v$ are solutions of the linear problems

$$
\begin{aligned}
& \left(\frac{\partial}{\partial t}+\frac{\partial}{\partial x}\right) u=0, u(x, 0)=f(x), \\
& \left(\frac{\partial}{\partial t}-\frac{\partial}{\partial x}\right) v=0, v(x, 0)=g(x),
\end{aligned}
$$

where $f, g \in \widehat{L^{p}}, 1<p<\infty$, the following estimate holds:

$$
\|u v\|_{\widehat{L^{p}}} \leq c\|f\|_{\widehat{L^{p}}}\|g\|_{\widehat{L^{p}}}
$$


Proof. We have $u(x, t)=f(x-t)$ and $v(x, t)=g(x+t)$ and thus $\tilde{u}(\xi, \tau)=$ $\delta(\tau+\xi) \widehat{f}(\xi)$ and $\tilde{v}(\xi, \tau)=\delta(\tau-\xi) \widehat{g}(\xi)$. This implies

$$
\begin{aligned}
\mathcal{F}(u v)(\xi, \tau) & =\iint \delta(\lambda+\eta) \widehat{f}(\eta) \delta(\tau-\lambda-(\xi-\eta)) \widehat{g}(\xi-\eta) d \lambda d \eta \\
& =\iint \delta(\tau+2 \eta-\xi) \widehat{f}(\eta) \widehat{g}(\xi-\eta) d \eta \\
& =\widehat{f}\left(\frac{\xi-\tau}{2}\right) \widehat{g}\left(\frac{\xi+\tau}{2}\right) .
\end{aligned}
$$

Consequently

$$
\|u v\|_{\widehat{L^{p}}}=\left(\iint\left|\widehat{f}\left(\frac{\xi-\tau}{2}\right) \widehat{g}\left(\frac{\xi+\tau}{2}\right)\right|^{p^{\prime}} d \xi d \tau\right)^{1 / p^{\prime}} \leq c\|f\|_{\widehat{L^{p}}}\|g\|_{\widehat{L^{p}}} .
$$

This proposition implies

Corollary 2.1. Let $1<p<\infty, \sigma>1 / p$. Then

$$
\|u v\|_{\widehat{L^{p}}} \leq c\|u\|_{X_{+p}^{0, \sigma}}\|v\|_{X_{-p}^{0, \sigma}} .
$$

Proof. Recall that (with $U_{ \pm}(t)$ denoting the evolution operator of the equation $\left.\left(\frac{\partial}{\partial t} \pm \frac{\partial}{\partial x}\right) u=0\right)$ :

$$
\|u\|_{X_{ \pm p}^{0, \sigma}}=\left(\int\langle\tau\rangle^{\sigma p^{\prime}}\left\|\mathcal{F}\left(U_{ \pm}(-\cdot) u\right)(\xi, \tau)\right\|_{L_{\xi}^{p^{\prime}}}^{p^{\prime}} d \tau\right)^{1 / p^{\prime}} .
$$

We use

$$
u(t)=c \int e^{i t \tau} U_{+}(t) h(\tau) d \tau \quad \text { with } \quad h:=\mathcal{F}_{t} U_{+}(-\cdot) u
$$

and

$$
v(t)=c \int e^{i t \rho} U_{-}(t) l(\rho) d \rho \quad \text { with } \quad l:=\mathcal{F}_{t} U_{-}(-\cdot) u .
$$

Thus

$$
(u v)(t)=c \iint e^{i t \tau} U_{+}(t) h(\tau) e^{i t \rho} U_{-}(t) l(\rho) d \tau d \rho .
$$

By Minkowski's inequality

$$
\|u v\|_{\widehat{L_{x t}^{p}}} \leq c \iint\left\|e^{i t(\tau+\rho)} U_{+}(t) h(\tau) U_{-}(t) l(\rho)\right\|_{\widehat{L_{x t}^{p}}} d \tau d \rho .
$$

But now for fixed $\tau$ and $\rho$ we have

$$
\begin{aligned}
\left\|e^{i t(\tau+\rho)} w(x, t)\right\|_{\widehat{L_{x t}^{p}}} & =\left\|\mathcal{F}\left(e^{i t(\tau+\rho)} w(x, t)\right)\right\|_{L_{\xi \tau^{\prime}}^{p^{\prime}}} \\
& =c\left\|\iint e^{-i\left(x \xi+t\left(\tau^{\prime}-\tau-\rho\right)\right)} w(x, t) d x d t\right\|_{L_{\xi \tau^{\prime}}^{p^{\prime}}}, \\
& =c\left\|\tilde{w}\left(\xi, \tau^{\prime}-\tau-\rho\right)\right\|_{L_{\xi \tau^{\prime}}^{p^{\prime}}}=c\left\|\tilde{w}\left(\xi, \tau^{\prime}\right)\right\|_{L_{\xi \tau^{\prime}}^{p^{\prime}}}=c\|w\|_{\widehat{L_{x t}^{p}}},
\end{aligned}
$$


so that with $w(t)=U_{+}(t) h(\tau) U_{-}(t) l(\rho)$ we get by Proposition 2.3:

$$
\begin{aligned}
\|u v\|_{\widehat{L_{x t}^{p}}} \leq c \iint\left\|U_{+}(t) h(\tau) U_{-}(t) l(\rho)\right\|_{\widehat{L_{x t}^{p}}} d \tau d \rho \\
\leq c \int\|h(\tau)\|_{\widehat{L_{x}^{p}}} d \tau \int\|l(\rho)\|_{\widehat{L_{x}^{p}}} d \rho \\
=c \int\langle\tau\rangle^{-\sigma}\langle\tau\rangle^{\sigma}\left\|\mathcal{F}\left(U_{+}(-\cdot) u\right)\right\|_{L_{\xi}^{p^{\prime}}} d \tau \int\langle\rho\rangle^{-\sigma}\langle\rho\rangle^{\sigma}\left\|\mathcal{F}\left(U_{-}(-\cdot) v\right)\right\|_{L_{\xi}^{p^{\prime}}} d \rho \\
\leq c\left(\int\langle\tau\rangle^{-\sigma p} d \tau\right)^{1 / p}\left(\int\langle\tau\rangle^{\sigma p^{\prime}}\left\|\mathcal{F}\left(U_{+}(-\cdot) u\right)\right\|_{L_{\xi}^{p^{\prime}}}^{p^{\prime}} d \tau\right)^{1 / p^{\prime}} \cdot \\
\quad \cdot\left(\int\langle\rho\rangle^{-\sigma p} d \rho\right)^{1 / p}\left(\int\langle\rho\rangle^{\sigma p^{\prime}}\left\|\mathcal{F}\left(U_{-}(-\cdot) v\right)\right\|_{L_{\xi}^{p^{\prime}}}^{p^{\prime}} d \rho\right)^{1 / p^{\prime}} \\
\leq c\|u\|_{X_{+p}^{0, \sigma}\|v\|_{X_{-p}^{0, \sigma}} .}
\end{aligned}
$$

We also used the following elementary algebraic inequality for real numbers.

Lemma 2.1. If $\xi_{1}, \xi_{2}, \tau_{1}, \tau_{2} \in \mathbf{R}$ and $\xi=\xi_{1}+\xi_{2}, \tau=\tau_{1}+\tau_{2}$, the following estimate holds:

$$
\min \left(\left|\xi_{1}\right|,\left|\xi_{2}\right|\right) \leq 1 / 2\left(\left|\sigma_{ \pm}\right|+\left|\sigma_{1}^{+}\right|+\left|\sigma_{2}^{-}\right|\right),
$$

where

$$
\sigma_{ \pm}:=\tau \pm|\xi|, \sigma_{1}^{+}:=\tau_{1}+\xi_{1}, \sigma_{2}^{-}:=\tau_{2}-\xi_{2}
$$

Proof. We have

$$
\sigma_{ \pm}=\tau \pm|\xi|=\sigma_{1}^{+}+\sigma_{2}^{-} \pm\left|\xi_{1}+\xi_{2}\right|-\xi_{1}+\xi_{2} .
$$

Now, if $\xi_{1}+\xi_{2} \gtreqless 0$, we have $\pm\left|\xi_{1}+\xi_{2}\right|-\xi_{1}+\xi_{2}=2 \xi_{2}$, whereas, if $\xi_{1}+\xi_{2} \leq 0$ we have $\pm\left|\xi_{1}+\xi_{2}\right|-\xi_{1}+\xi_{2}=-2 \xi_{1}$, so that the claimed inequality follows.

\section{References}

[AFS] P. D'ancona, D. Foschi and S. Selberg, Null structure and almost optimal local regularity for the Dirac - Klein - Gordon system. arXiv: math. $\mathrm{AP} / 0509545$, to appear in Journal of the EMS.

[B] N. Bournaveas, A new proof of global existence for the Dirac Klein-Gordon equations in one space dimension, J. Funct. Analysis 173 (2000), 203-213.

[BG] N. Bournaveas and D. Gibbeson, Low regularity global solutions of the Dirac - Klein - Gordon equations in one space dimension, Diff. Int. Equations 19 (2006), 211-222.

[CVV] T. Cazenave, L. Vega and M. C. Vilela, A note on the nonlinear Schrödinger equation in weak $L^{p}$ spaces, Comm. Contemp. Math. 3 (2001), 153-162. 
[C] J. M. Chadam, Global solutions of the Cauchy problem for the (classical) coupled Maxwell - Dirac equations in one space dimension, J. Funct. Analysis, 13 (1973), 173-184.

[CG] J. M. Chadam and R. T. Glassey, On certain global solutions of the Cauchy problem for the (classical) coupled Klein - Gordon - Dirac equations in one and three space dimensions, Arch. Rat. Mech. Anal. 54 (1974), 223-237.

[F] Yung-Fu Fang, A direct proof of global existence for the Dirac - Klein Gordon equations in one space dimension, Taiwanese J. Math. 8 (2004), 33-41.

[F1] Yung-Fu Fang, Low regularity solutions for Dirac - Klein - Gordon equations in one space dimension, Electr. J. Diff. Equ. 2004(102) (2004), 1-19.

[G1] A. Grünrock, An improved local well-posedness result for the modified $K d V$ equation, Int. Math. Res. Not. (61) (2004), 3287-3308.

[G2] A. Grünrock, Bi- and trilinear Schrödinger estimates in one space dimension with applications to cubic NLS and DNLS. Int. Math. Res. Not. (41) (2005), 2525-2558.

[M] S. Machihara, The Cauchy problem for the 1d Dirac-Klein-Gordon equation. to appear in NoDEA

[P] H. Pecher, Low regularity well-posedness for the one-dimensional DiracKlein-Gordon system, Electr. J. Diff. Equ. 2006(150) (2006), 1-13.

[ST] S. Selberg and A. Tesfahun, Low regularity well-posedness for the one dimensional Dirac-Klein-Gordon system, arXiv: math. AP/0611718

[VV] A. Vargas and L. Vega, Global wellposedness for $1 D$ non-linear Schrödinger equation for data with infinite $L^{2}$ norm. J. Math. Pures Appl. 80 (2001), 1029-1044.

Hartmut Pecher

Fachbereich Mathematik und Naturwissenschaften

Bergische Universität Wuppertal

Gaußstr. 20

42097 Wuppertal

Germany

e-mail: Hartmut.Pecher@math.uni-wuppertal.de

Received: 4 May 2007

Accepted: 28 September 2007

Published Online First 03 October 2008

To access this journal online:

http://www.birkhauser.ch 